\title{
Matrimonio infantil: Un problema social, económico y de salud pública
}

\author{
Luis Alfonso Mendoza Tascón 1,2, Diana Isabel Claros Benítez², Laura Isabel Mendoza \\ Tascón², Claudia Bibiana Peñaranda Ospina1, Martha Deyfilia Arias Guatibonza1, \\ Jorge Hernán Carrillo Bejarano ${ }^{1}$, Zoolange Sarria Henao. ${ }^{1}$ \\ ${ }^{1}$ Fundación Hospital San José de Buga. Buga, Colombia. ${ }^{2}$ Facultad Ciencias de la Salud, Programa de Medicina, Unidad \\ Central del Valle del Cauca. Tuluá, Colombia.
}

\section{RESUMEN}

Matrimonio infantil se define como cualquier unión legal o unión habitual entre dos personas, de las cuales una o ambas están por debajo de los 18 años de edad. Sus efectos no solo comprometen la salud de la niña, sino que repercute en lo social, lo económico y en el desarrollo de las comunidades. Las prevalencias de matrimonio infantil son altas en los países con menos desarrollo y más pobres. Sus consecuencias abarcan involucran lo social dado que el el matrimonio infantil es una violación de los derechos de los niños, afectando y limitando su educación, lo que se traduce en menores oportunidades laborales, afectando la economía de los hogares y las comunidades, comprometiendo la salud de las niñas y sus hijos y asociado a violencia intrafamiliar. Las estrategias de prevención son múltiples e involucran la capacitación de las niñas con la información, las habilidades y las redes de apoyo, la educación y movilización de padres y miembros de la comunidad, mejora de la accesibilidad y calidad de la educación formal para las niñas, el ofrecimiento de apoyo económico e incentivos para niñas y sus familias y el fomento de un marco jurídico y normativo favorable.

\section{PALABRAS CLAVE: Niñez, adolescencia, matrimonio infantil, educación}

\section{SUMMARY}

Child marriage is defined as any union or usual legal union between two people, of which one or both are below 18 years of age. Its effects not only compromise the health of the child, it affects social, economic and community development. The prevalence of child marriage is high in countries with less developed and poorer. Its consequences include social as involving the child marriage is a violation of the rights of children, affecting and limiting their education, resulting in lower labor opportunities, affecting the economy of households and communities, jeopardizing health girls and their children and associated with domestic violence. Prevention strategies are multiple and involve the training of girls with the information, skills and support networks, education and mobilization of parents and community members, improving accessibility and quality of formal education for girls, offering financial support and incentives to girls and their families and the promotion of an enabling legal and regulatory framework.

KEY WORDS: Childhood, adolescence, child marriage, education 


\section{INTRODUCCIÓN}

Los Objetivos para el Desarrollo del Milenio (ODM) articulan un conjunto de objetivos para motivar y acelerar el progreso global en lo social, económico, en el desarrollo y la sostenibilidad ambiental. Ocho ODM se centran en acabar con la pobreza y el hambre, lograr la educación primaria universal, promover la igualdad de género, mejorar la supervivencia infantil y la salud materna, reducción del VIH/SIDA, tuberculosis y malaria, garantizar la sostenibilidad del medio ambiente, promocionar un entorno mundial propicio para el desarrollo con respecto a asuntos internacionales, como las relaciones económicas, financieras y comerciales.

Los objetivos cuarto y quinto hacen referencia a la supervivencia infantil y la salud materna, respectivamente. El segundo objetivo del ODM, es el logro del acceso universal a la salud reproductiva para el año 2015. El progreso hacia ese objetivo se mide a través de cuatro indicadores: 1. Tasa de uso de anticonceptivos, 2. Proporción de la demanda de planificación familiar que es insatisfecha, 3 . Tasa de nacimientos en adolescentes y proporción de mujeres embarazadas que reciben cuidados de atención prenatal (1). La salud sexual y reproductiva (SSR) de la adolescente es una de las cinco áreas del Plan de Acción para la Salud Reproductiva del Banco Mundial 2010-2015, que reconoce la importancia de abordar este tema, como un problema de desarrollo con implicaciones importantes para la reducción de la pobreza (2).

Las intervenciones preconcepcionales que buscan mejorar los resultados maternos y perinatales, incluyen mejoramiento de la SSR de las mujeres mediante la planificación familiar, para evitar embarazos no deseados, optimizar el intervalo entre embarazos, el tratamiento de las infecciones de transmisión sexual (ITS), incluyendo el VIH (3).

La SSR de las adolescentes es de particular importancia debido a los mayores riesgos de muerte fetal, parto prematuro, bajo peso al nacer, asfixia, mortalidad materna asociada al embarazo adolescente (4). En contextos en los que el embarazo adolescente ocurre normalmente dentro del matrimonio, las intervenciones y programación de políticas para prevenir el matrimonio infantil, promover la educación, empoderar a las niñas y el abordaje de normas socioculturales dirigidas a mejorar la SSR, deben estar en la agenda (5).

\section{DEFINICIONES}

Niño: Toda persona, mujer u hombre, menor de 18 años de edad $(6,7)$.

Adolescencia: La adolescencia según la OMS es el período de la vida comprendido entre los 10 y 19 años (8-13) dividido en tres etapas: 1. Adolescencia temprana (10-14 años), 2. Adolescencia media (15-16 años) y 3. Adolescencia tardía (17-19 años) (8-14).

Matrimonio infantil: Se define como cualquier unión legal o unión habitual entre dos personas, de las cuales una o ambas están por debajo de 18 años de edad. Esta definición se basa en varias convenciones, tratados y acuerdos internacionales, entre ellos la Convención sobre la Derechos del Niño, Convención sobre la eliminación de todas las formas de discriminación contra la Mujer, Declaración Universal de Derechos Humanos, y las recientes resoluciones de la ONU Consejo de Derechos Humanos $(6,15)$.

\section{MAGNITUD DEL PROBLEMA}

El matrimonio antes de los 18 años es una violación de los derechos fundamentales, afectando de manera más desproporcionada a mujeres que a varones. En todo el mundo, más de 60 millones de mujeres de 20-24 años se han casaron antes de llegar a los 18 años, siendo las tasas más altas en África Occidental, seguido por el sur de Asia, norte de África, Oriente Medio y América Latina y El Caribe (ALC) (16). Si las tendencias actuales continúan, más de 142 millones de niñas menores de 18 años estarán casadas en la próxima década, es decir 39.000 niñas cada día contraerán matrimonio $(6,17)$. El matrimonio infantil en países en desarrollo sigue siendo generalizada, donde una tercera parte de las niñas se casan antes de los 18 años de edad y 1 de cada 9 están casadas antes de los 15 años de edad (18).

La prevalencia regional del matrimonio infantil oscila entre $21 \%$ en África, $17 \%$ en ALC, a $8 \%$ en el Este y Sur de Europa. La prevalencia para algunas regiones oscila entre $2 \%$ en Argelia y Libia, hasta un máximo de 74-75\% en Niger y Bangladesh (19). En ALC, Guyana, Surinam, Ecuador y Paraguay el $11-20 \%$ de sus mujeres de 20-24 años se han casado antes de los 18 años de edad, el $21-30 \%$ en México, Guatemala, Belice, Colombia, Ecuador y Bolivia y el 36-40\% en Brasil (20).

La cohabitación es una parte integral de la investigación de la familia. Sin embargo, pocos trabajo evalúan la cohabitación entre los adolescentes o niños, o los vínculos entre la convivencia y la maternidad adolescente. Un 34\% de adolescentes conviven, se casan o tienen un hijo. La gran mayoría de las adolescentes embarazadas solteras no forman una unión antes del nacimiento de su hijo y sólo $22 \%$ cohabitan y $5 \%$ se casan. Sin embargo, las adolescentes embarazadas más individuales finalmente cohabitan, 59\% lo hace en el tercer cumpleaños del hijo y $9 \%$ se casan (21). 


\section{FACTORES DETERMINANTES DEL MATRIMO- NIO INFANTIL Y EN ADOLESCENTES}

Son varios los determinantes que se han asociado con el matrimonio infantil y del adolescente, incluyendo normas sociales y culturales, situación socioeconómica, niveles de educación y contexto de la comunidad. Hay sociedades donde los padres deciden sobre el matrimonio de sus hijas por intereses o necesidades económicas. Las niñas que conviven en hogares pobres, con menor conocimiento de los impactos del matrimonio infantil, que son 5 o más años menores que sus actuales esposos o de zona rural, son más propensas a contraer matrimonio antes de los 18 años $(22,23)$. En otras sociedades, hay tradiciones locales como por ejemplo en Bangladesh, República de Kirguistán y Somalia, donde obligan a las niñas que han sido secuestradas y violadas a casarse con el agresor para proteger el honor de sus familias (24). El matrimonio adolescente es también mucho más probable que afecte a mujeres que hombres. En el mundo en desarrollo, $16 \%$ de mujeres están casadas en comparación con el $3 \%$ de varones (25).

Un análisis de los datos de seis países (Bangladesh, Burkina Faso, Etiopia, Nepal, Niger, Nigeria) mostró que la actividad sexual (AS) de adolescentes está estrechamente ligada al estado civil. Se encontró que casi todas las mujeres adolescentes casadas alguna vez han tenido AS, mientras que las no casadas han tenido abstinencia sexual. La edad a la primera relación sexual también está estrechamente relacionada con la edad al matrimonio. En Nigeria, Bangladesh y Nigeria, más de un tercio de las mujeres casadas alguna vez tuvieron relaciones sexuales antes de los 15 años (2).

Algunas niñas y adolescentes que deciden casarse, utilizan el matrimonio como una manera de satisfacer su necesidad de libertad, experimentar el amor en un marco culturalmente respetable, escapar de la pobreza y de sus familias cuando hay violencia o su vida es difícil. Para algunas, sin embargo, el matrimonio precoz ocasiona que estas no alcancen sus objetivos académicos, sienten intensa soledad y tengan problemas económicos (26).

Un análisis realizado a la base de datos de National Youth in Transition Database (n: 15.601) informa que $4 \%$ de varones y $10 \%$ de mujeres tienen hijos, donde para ambos, la raza no blanca y el origen hispano se asociaron con mayor riesgo de parto. Para los hombres, la probabilidad de ser padre aumentan si el adolescente tiene historia de falta de vivienda, abuso de sustancias psicoactivas o se encuentra encarcelado (27). En países como Uganda, Tanzania y Kenia, la proporción de nacimientos en mujeres menores de 20 años es de $57 \%, 56 \%$ y $47 \%$ respectivamente, con mayor proporción entre los 18-19 años, seguido de 16-17 años y menores de 16 años. En estos países, en- tre un $7,2-12,3 \%$ de mujeres menores de 16 años tienen hijos. La mayor proporción de estas mujeres viven en zona rural, sin embargo, entre las menores de 16 años con hijos, la mayoría vive en el área urbana. Gran parte de estas mujeres son más pobres, carentes de educación o sólo con educación primaria, sobre todo las menores de 16 años. Una mayor proporción de mujeres adolescentes se casan en el momento del primer parto (Uganda $75 \%$, Tanzania $72 \%$ y Kenia $57 \%$ ), siendo mayor el número de matrimonios en el área rural y a mayor edad de la mujer. En estos países el matrimonio o la unión consensuada, puede ser impulsada por el embarazo y no al contrario, dado que la actividad sexual prematrimonial es elevada (28).

\section{IMPACTO DEL MATRIMONIO DE NIÑOS Y ADO- LESCENTES}

Social: En la 65 a Asamblea Mundial de la Salud, los representantes coincidieron en que el matrimonio infantil es una violación de los derechos de los niños y adolescentes, siendo ilegal en la mayoría de los lugares donde se produce. Limita la autonomía de las niñas, el conocimiento, los recursos y el poder de decisión (29). El matrimonio infantil sitúa a la niña bajo el control de su marido, y a veces de las leyes, limitando su capacidad para expresar sus opiniones, para formar y llevar a cabo sus propios planes y aspiraciones. El matrimonio de las niñas las aísla de la escuela, amigos y de las oportunidades de trabajo, obstaculiza su acceso al apoyo social que es importante para su bienestar emocional, y limita su acceso al capital social y redes que pueden aumentar sus ingresos económicos. Incluso, muchas pierden o carecen de capacidad para negociar la actividad sexual, el uso de anticonceptivos o espaciar el nacimiento de sus hijos. Muchas tienen restricciones incluso de su tiempo, lo cual además de restringir la educación, también las restringe para acceder a información y servicios de salud, incluida la mental, afectando su seguridad y autoestima. Las mujeres que no pueden tomar decisiones sobre ellas o su hogar, van a tener problemas en la crianza de sus hijos (30).

Educación: Cuando una niña se casa, la trayectoria educativa cambia o cesa, deteniendo la adquisición de conocimientos y habilidades para su vida, reduciendo ingresos económicos, dejando incluso de ser un miembro más productivo para su familia y comunidad. Esto se convierte en barrera para la adquisición de un empleo formal remunerado (30). Estas mujeres pierden la red social y la estructura de soporte que las escuelas proporcionan, impidiendo participar en asuntos y ocupaciones comunitarias (31). Por otra parte, la educación en escuelas les permite a las niñas acceder a informar valiosa en temas de salud, principalmente en SSR, mejorando el uso de los recursos y acceso a los 
servicios de salud, afectando la salud de sus propios hijos $(32,33)$.

Participación laboral: Las niñas y adolescentes que se casan a temprana edad, ocupan gran parte de su tiempo en trabajo doméstico no remunerado, con fertilidad más elevada, maternidad más temprana, períodos intergésicos cortos, mayor número de hijos, lo que limita su educación, su rendimiento en la fuerza laboral, repercutiendo en el crecimiento económico de las comunidades (30).

Salud: La AS es un factor clave que contribuye a la SSR de niños/as y adolescentes. La AS premarital y de inicio temprano, pone a niños/as y adolescentes, principalmente a mujeres, en mayor riesgo de embarazos no deseados, infecciones de transmisión sexual (ITS), infección por HIV/SIDA, morbilidad y mortalidad maternas y de sus hijos (34-38).

El embarazo en la niñez y adolescencia se asocia a morbilidades y graves complicaciones (físicas, psicológicas y sociales), que requieren de mayor tiempo para su recuperación, afectando la economía de sus familias y comunidades (39).

La investigación ha demostrado que el matrimonio de adolescentes se asocia con embarazo, con embarazo no planificado, rápida repetición de parto, uso inadecuado de los servicios de salud materna y pobres resultados del parto, entre otros resultados de salud materna e infantil negativos (19,40-42). Por ello, el retraso de la maternidad y la prevención de embarazos no deseados en la niñez y adolescencia, se ha demostrado que mejora los resultados de salud y aumenta las oportunidades de educación, empleo en el futuro, y las ganancias $(43,44)$.

El riesgo de muerte asociado con el embarazo es alrededor de un tercio más alta entre los 15-19 años que entre los 20-24 años de edad (45). En 2015, se estimó que 303.000 mujeres murieron por causas relacionadas con el embarazo o el parto (la mayoría de ellos murieron a causa de hemorragias graves, sepsis, eclampsia, parto obstruido y las consecuencias de los abortos inseguros). El problema no para allí, cuando las madres mueren, sus familias son mucho más vulnerables y sus hijos tienen más probabilidades de morir antes de cumplir los dos años. (46) Althabe y cols (47), encontraron un mayor riesgo de parto prematuro y bajo peso al nacer entre las adolescentes de 15-19 años (mayor en las menores de 15 años de edad), pero no de hemorragia preparto, hemorragia postparto, parto obstruido, sepsis, mortalidad fetal in útero, ni mortalidad neonatal a los 28 días cuando se les comparó con las de 20-24 años.

La creciente evidencia del África subsahariana muestra que las niñas que se casan tempranamente, tienen mayor riesgo de contraer el VIH u otra ITS. El matrimonio antes de los 20 años se considera un factor de riesgo para la infección por VIH. En Kenia y Zambia, las tasas de infección por VIH son mayores entre niñas casadas que solteras (24). En Uganda la prevalencia de infección por VIH en mujeres entre 15-19 años de edad es mayor para las casadas $(89 \%)$ que para las solteras $(66 \%)(48,49)$. siendo la diferencia de edad entre sus conyugues un factor de riesgo significativo.

Violencia: El matrimonio infantil se puede considerar una de las formas de violencia contra las niñas. Con frecuencia hay una gran diferencia entre las niñas y sus maridos. Investigaciones realizadas en Camboya, Colombia, Haití, India, Kenia, Perú, Sudáfrica, Turkmenistán y Zambia, sugieren que el tener un marido de mayor edad aumenta dramáticamente el riesgo de violencia de pareja (24). Las niñas y adolescentes que se casan antes de los 15 años tienen un mayor riesgo de violencia en la pareja y el sexo coercitivo comparado con las que casan entre 15-18 años de edad. Las mujeres que se casan después de los 18 años tienen mayor probabilidad de rechazar las relaciones sexuales (50).

\section{PREVENCIÓN DEL MATRIMONIO A TEMPRANA EDAD}

Para prevenir el matrimonio infantil o muy temprano en la adolescencia, se deben evaluar a profundidad las causas y las consecuencias (30). Existen más de 150 intervenciones (23 de ellas documentaron intento de medir cambios en los comportamientos relacionados con el matrimonio infantil) para la prevención del matrimonio infantil. La mayoría de los programas trabajan directamente con niñas, a quienes se les ofrece oportunidades para obtener habilidades y educación. La mayoría de los esfuerzos involucran a familias y miembros de la comunidad para tratar de cambiar las normas sociales subyacentes que perpetúan la práctica del matrimonio infantil. Muchas intervenciones también ofrecen incentivos económicos a los padres para promover la educación y conductas saludables, así como prevenir el matrimonio infantil. La evaluación de estos 23 programas, identificó cinco estrategias principales para prevenir o retrasar el matrimonio infantil (Tabla I) (16). Estos programas deben ser evaluados con la finalidad de conocer los cambios en los conocimientos, actitudes y comportamientos en relación con el matrimonio infantil entre las niñas en situación de riesgo, padres, miembros y funcionarios de la comunidad. Las medidas de éxito deben incluir: 1. Aumento en el conocimiento de las consecuencias negativas relacionadas con el matrimonio infantil, 2. Aumento de la edad ideal para contraer matrimonio para las niñas, 3. Cambio en actitudes y apoyo a las niñas, para que tomen las decisiones respecto a su matrimonio, 4. Aumento de la edad de matrimonio para las niñas y niños, y 5. Proporciones más pequeñas de niñas se casan antes de los 18 años de edad. 
Tabla I

\section{ESTRATEGIAS PRINCIPALES PARA RETRASAR EL MATRIMONIO O PREVENIR EL MATRIMONIO INFANTIL}

Estrategia
1. Capacitar a las niñas con la información, las habili-
dades y las redes de apoyo

2. La educación y la movilización de los padres y miembros de la comunidad

3. Mejora de la accesibilidad y calidad de la educación formal para las niñas

4. Ofrecer apoyo económico y los incentivos para las niñas y sus familias

5. Fomentar un marco jurídico y normativo favorable

\section{Características}

Concentrado en las niñas, con un programa centrado en la formación, el desarrollo de habilidades, el intercambio de información, la creación de espacios seguros y el desarrollo de redes de apoyo. La razón principal detrás de este conjunto de intervenciones es para equipar a las jóvenes para que conozcan mejor a sí mismos, su mundo y sus opciones y para poner fin a su aislamiento social y económico, lo que les permite actuar y abogar por ellos mismos, tanto a corto como a largo plazo.

La principal motivación detrás de esta estrategia para "crear un entorno propicio" en el entendimiento de que la decisión de casarse con niñas, está generalmente en manos de los adultos de la familia y de la comunidad, y que da como resultado la estigmatización y las sanciones por no cumplir con las expectativas sociales, que son administradas por la comunidad en general.

La educación además de mejorar las probabilidades laborales, sirve de protección contra el matrimonio por lo menos por varias razones. El hecho de estar en escuela, las ayuda a ser tratadas como niñas y por lo tanto a no casarse. Aparte del hogar, las escuelas pueden ser vistas como un "espacio seguro" para las niñas. Además, la experiencia y el contenido de la educación, ayuda a las niñas a desarrollar redes sociales y a adquirir habilidades e información, las cuales contribuyen a su capacidad de comunicarse mejor y negociar sus intereses.

Los fundamentos de este enfoque, es que las oportunidades económicas inmediatas proporcionarían una alternativa aceptable frente al matrimonio y aumentan la sensación de seguridad y crecimiento económico. Esto reduce la presión económica y social para casar o casarse con una niña. Estos incentivos financieros a veces están ligados a inversiones educativas en las hijas y/o de la condición que no se casan hasta los 18 años.

Muchos países con altas tasas de matrimonio infantil tienen leyes que prohíben esta práctica, y realizan grandes esfuerzos políticos para fortalecer y/o hacer cumplir esas leyes. Las iniciativas políticas y jurídicas presentan algunos desafíos, ya que la aplicación universal es difícil. A veces se hace difícil diferenciar estas iniciativas impulsadas desde lo legal y desde lo político.
Aunque no es suficiente por sí solo, las reformas legales son importantes. La reforma del Derecho y un marco político se hace necesaria. Se requieren intervenciones que promuevan una mayor comunicación con equidad de género y la toma de decisiones en torno a la SSR, así como mejorar la calidad y el acceso de las mujeres a los servicios de salud. Hay intervenciones que incluyen incentivos a niñas y sus familias para que mantengas las niñas en escuelas y eviten el matrimonio antes de los 18 años, mejorando las oportunidades de educación para ellas. Se hace necesario involucrar a los hombres, las comunidades y las autoridades tradicionales para cambiar la cultura y las normas entorno a la sexualidad, el matrimonio y la reproducción (51). En los hogares, se deben repartir las responsabilidades entre hombres y mujeres. Igualmente, se debe educar a los varones en SSR, así 
como en anticoncepción (52), esto facilita la comunicación entre las parejas de esposos y resulta en la mejor toma de decisiones (53).

Un aspecto muy importante es la mejora de los servicios de salud, donde se brinde más educación a parejas y familias en temas de SSR y anticoncepción. Se debe mejorar la calidad de la educación, con lo cual habrá menos deserción escolar, lo cual distancia la edad del matrimonio de las niñas. Definitivamente, la educación con niñas, adolescentes, padres de familia y comunidad es la mejor estrategia para prevenir el matrimonio infantil. Los enfoques multisectoriales son probablemente más eficaces que una sola intervención, debido a que el embarazo temprano tiene muchos factores determinantes entrelazados, incluyendo la falta de educación, la falta de oportunidades, además de los problemas económicos (24).

\section{CONCLUSIONES}

El matrimonio infantil es un grave problema social, con repercusiones económicas y para salud para los niños/as, sus familias y las comunidades. Parece necesario implementar algunas intervenciones preventivas específicas, incluyendo los programas de educación para aumentar la conciencia pública acerca de las consecuencias sociales, físicos y fiscales de los matrimonios precoces. Sin embargo, las intervenciones más efectivas constituyen aquellas donde se empodera a las niñas con información, con habilidades para empoderar a las niñas para tomar decisiones sobre su vida, con redes de apoyo y mejoramiento de la accesibilidad y educación formal, oferta de apoyo económico e incentivos para niñas y sus familias, para mantenerlas en la escuela y que se casen más tardíamente. Se requiere igualmente, la movilización de la comunidad en la oposición del matrimonio infantil.

\section{REFERENCIAS}

1. Gaffey MF, Das JK, Bhutta ZA. Millennium Development Goals 4 and 5: Past and future progress. Semin Fetal Neonat Med 2015;20:285-92.

2. Cortéz R, Yarger J, Decker M, Brindis C. Health, Nutrition and Population Global Practice of the World Bank Group. January 2015. Socioeconomic differences in adolescent sexual and reproductive health: sexual activity. Acceso: 09-01-2015. Disponible en: http://www-wds.worldbank.org/external/default/WDSContentServer/WDSP/IB/2015/01/29/000333037_20 150129023454/Rendered/PDF/935430REVISED00vi ty0JAN.26.150FINAL.pdf

3. Lassi Z, Mansoor T, Salam RA, Das JK, Bhutta ZA. Essential pre-pregnancy and pregnancy interventions for improved maternal, newborn and child health. Reprod Health 2014;11(Suppl.1):S2.

4. Bhutta ZA, Das JK, Bahl R, Lawn JE, Salam RA, Paul VK, et al. Can available interventions end preventable deaths in mothers, newborn babies, and stillbirths, and at what cost? Lancet 2014;384(9940):347-70.

5. Partnership for Maternal, Newborn and Child Health (PMNCH). Knowledge Summary 22: reaching child brides. PMNCH; 2012. Acceso: 10-01-2016. Disponible: http://portal.pmnch.org/ knowledge-summaries/ ks22.

6. UNICEF (United Nations Children's Fund). 2014a. Ending Child Marriage: Progress and Prospects. New York: UNICEF.

7. UNICEF. Convención de los derechos del niño. Acceso: 13-01-2016. Disponible: http://www.eird.org/cd/ toolkit08/material/otros/derechos_de_los_ninos/articulos.pdf.

8. Issler JR. Embarazo en la adolescencia. Revista de posgrado de la VI cátedra de Medicina 2001;107:1123.

9. Serra FG, Spaciuk SM, Morales E, Arce GM, Avalos ME, Gómez VY. Riesgo reproductivo del adolescente en Corrientes. Revista de posgrado de la VI cátedra de Medicina 2002;115:24-5.

10. Balestena JM, Balestena SG. Impacto de la menarquía en los resultados maternos perinatales en la adolescencia. RCOG [en línea] 2005 [2 de enero de 2012];31(1).URL disponible en: http://www.bvs.sld.cu/ revistas/gin/vol31_1_05/gin02105. htm.

11. Laffita A, Ariosa JM, Cutié JR. Adolescencia e interrupciones del embarazo. RCOG [en línea] 2004 [2 de enero de 2012];30(1).URL disponible en: http://www. bvs.sld.cu/revistas/gin/vol30_1_04/gin04104.htm.

12. Valdés S, Essien J, Bardales J, Saavedra D, Bardales $\mathrm{E}$. Embarazo en la adolescencia. Incidencia, riesgos y complicaciones. RCOG. [en línea] 2002 [2 de enero de 2012 de noviembre de 2005]; 28(2). URL disponible en: http://www.bvs.sld.cu/revistas/gin/vol28_2_02/ gin04202.htm.

13. Fernández LS, Carro E, Osesa D, Pérez J. Caracterización del recién nacido en una muestra de gestantes adolescentes. RCOG [en línea] 2004 [2 de enero de 2012]; 30(2). URL disponible en: http://www.bvs.sld. $\mathrm{cu} / \mathrm{revistas} / \mathrm{gin} / \mathrm{vol} 30$ 2_04/gin03204.htm.

14. Romero MI, Maddaleno M, Silber TJ, Munist M. Salud reproductiva. En: Silber TJ, Munist MM, Maddaleno M, Suarez Ojeda. EN: Manual de medicina de la adolescencia. EEUU: Publicación de la OPS 1992:473518.

15. Scolaro E, Blagojevic A, Filion B, Chandra-Mouli V, Say L, Svanemy J, et al. Child Marriage Legislation in the Asia-Pacific Region. The Review of Faith \& International Affairs. 2015;13(3):23-31.

16. Malhotra A, Warner A, McGonagle $A$ and Lee-Rife S. 2011. Solutions to End Child Marriage What the Evidence Shows. Washington, DC: International Center for Research on Women (ICRW). Acceso: 14-012016. Disponible en: http://www.icrw.org/files/publications/Solutions-to-End-Child-Marriage.pdf.

17. UNFPA (United Nations Population Fund). (2012). Marrying Too Young: End Child Marriage. UNFPA: New York.

18. UNICEF, State of the World's Children (New York: UNICEF, 2013). Acceso: 14-01-2016. Disponible en: http://www.ncbi.nlm.nih.gov/pmc/articles/ PMC3394179/pdf/nihms347595.pdf

19. Raj A, Boehmer U. Child Marriage and Its Association with National Rates of HIV, Maternal Health, and Infant Mortality Across 97 Countries. Violence Against Women 2013;19(4):536-51. 
20. Voice and Agency 2014 team estimates based on Demographic and Health Surveys, International Center for Research on Women, and United Nations Children's Fund using latest available data from 20012012.

21. Wendy D, Manning WD, Cohen JA. Teenage Cohabitation, Marriage, and Childbearing. Population Research and Policy Review. 2015;34(2):161-77.

22. Papp SA, Gogoi A and Campbell C. "Can Social Accountability Improve Maternal Health in India?" London School of Economics and Political Science. Acceso 14-01-2016. Disponible en: http://blogs. Ise. ac.uk/indiaatlse/2013/11/06/cansocial-accountabilityinitiatives-improvematernal-health-in-india/

23. Heidari F, Dastgiri S. Prevalence and Determinants of Child Marriage. Razavi Inter J Med 2015;3(3):e15776.

24. The World Bank Group. 2014. Voice Agency and Empowering women and girls for shared prosperity. Acceso: 13-01-2016. Disponible en: http://www.worldbank.org/content/dam/Worldbank/document/Gender/ Voice_and_agency_LOWRES.pdf.

25. Loaiza E, Liang M. UNFPA (United Nations Population Fund). (2013). Adolescent Pregnancy: A Review of the Evidence. UNFPA: New York.

26. Segal-Engelchin D, Huss E, Massry N. The Experience of Early Marriage: Perspectives of Engaged and Married Muslim Women in Israel. J Adolesc Research 2015. Acceso: 13-01-2016. Disponible en: ar.sagepub. com/content/early/2015/09/15/0743558415605167.

27. Shpiegel S, Cascardi M. Adolescent Parents in the First Wave of the National Youth in Transition Database. Acceso: 13-01-2016. Disponible en: http://www. tandfonline.com/doi/full/10.1080/15548732.2015.104 3687.

28. Neal SE, Chandra-Mouli V, Chou D. Adolescent first births in East Africa: disaggregating characteristics, trends and determinants. Reprod Health 2015;12:13.

29. World Bank. 2014. Regional Reports from Latin America and the Caribbean, South Asia, East Asia and the Pacific, and West and Central Africa.

30. Parsons J, Edmeades J, Kes A, Petroni S, Sexton M, Wodon Q. Economic Impacts of Child Marriage. A Review of the Literature. The Review of Faith \& International Affairs 2015;13(3):12-22.

31. UNICEF (United Nations Children's Fund). 2014b. Hidden in Plain Sight: A Statistical Analysis of Violence Against Children. New York: UNICEF.

32. Abu-Ghaida D, Klasen S. The Costs of Missing the MiIlennium Development Goal on Gender Equity. World Development 2004;32(7):1075-7.

33. Boyle MH, Racine Y, Georgiades K, Snelling D, Hong $\mathrm{S}$, Omariba W, et al. The Influence of Economic Development Level, Household Wealth and Maternal Education on Child Health in the Developing World. Social Science \& Medicine 2006;63(8):2242-54.

34. Bearinger LH, Sieving RE, Ferguson J, Sharma V. Global Perspectives on the Sexual and Reproductive Health of Adolescents: Patterns, Prevention, and Potential. Lancet 2007;369(9568):1220-31.

35. Hindin MJ, Fatusi AO. Adolescent Sexual and Reproductive Health in Developing Countries: An Overview of Trends and Interventions. Inter Perspec Sex Reprod Health 2009;35(2):58-62.

36. Kaestle CE, Halpern CT, Miller WC, Ford CA. Young Age at First Sexual Intercourse and Sexually
Transmitted Infections in Adolescents and Young Adults. Am J Epidemiol 2005;161(8):774-80.

37. Prata N, Passano P, Sreenivas A, Gerdts CE. Maternal Mortality in Developing Countries: Challenges in Scaling-up Priority Interventions. Women's Health 2010;6(2):311-27.

38. Goli S, Rammohan A, Singh D. The Effect of Early Marriages and Early Childbearing on Women's Nutritional Status in India. Mater Child Health J 2015;19(8):186480.

39. Koblinsky M, Chowdhury ME, Moran A, Ronsmans C. Maternal Morbidity and Disability and their Consequences: Neglected Agenda in Maternal Health. J Health Popul Nutrit 2012;30(2):124-30.

40. Pradhan R, Wynter K, Fisher J. Factors associated with pregnancy among adolescents in low-income and lower middle-income countries: a systematic review. J Epidemiol Community Health 2015;69(9):91824.

41. Godha D, Hotchkiss DR, and Gage AJ. Association Between Child Marriage and Reproductive Health Outcomes and Service Utilization: A MultiCountry Study from South Asia. J Adolesc Health 2013;52(5):552-8.

42. Santhya KG. Early Marriage and Sexual and Reproductive Health Vulnerabilities of Young Women: A Synthesis of Recent Evidence from Developing Countries. Current Opinion Obstet Gynecol2011;23(5):334-9.

43. Greene ME, Merrick T. 2005. Poverty Reduction: Does Reproductive Health Matter? HNP Discussion Paper. Washington, DC: The World Bank.

44. UNFPA (United Nations Population Fund). Motherhood in childhood: Facing the challenge of adolescent pregnancy: The State of World Population 2013. New York: United Nations Population Fund; 2013:163-96.

45. Nove A, Matthews Z, Neal S, Camacho AV. Maternal mortality in adolescents compared with women of other ages: Evidence from 144 countries. Lancet Glob Health 2012;2:e155-64.

46. UNFPA (United Nations Population Fund). 2016. Maternal health. Significant strides, but not enough. New York: UNFPA. Acceso: 14-01-2016. Disponible en: http://www.unfpa.org/maternal-health.

47. Althabe F, Moore JL, Gibbons L, Berrueta M, Goudar $\mathrm{Sh}$, Chomba E, et al. Adverse maternal and perinatal outcomes in adolescent pregnancies: The Global Network's Maternal Newborn Health Registry study. Reprod Health 2015, 12(Suppl 2):S8.

48. World Bank. 2006. "Social Accountability: What Does It Mean for the World Bank?" In Social Accountability Sourcebook. Washington, DC: World Bank.

49. George A. Accountability in Health Services Transforming Relationships and Contexts. Indian Institute of Management and Harvard Center for Population and Development Studies, Working Paper 2013;13(1). Acceso: 16-01-2016. Disponible en: http://www.who.int/ management/partnerships/accountability/accountabilityhealthservicespdf.pdf.

50. Erulkar A. Early Marriage, Marital Relations and Intimate Partner Violence in Ethiopia. Int Perspect Sex Reprod Health 2013;39(1):6-13.

51. Fleming P, Barker G, Morton M, McCleary-Sills J. Engaging Men and Boys in Advancing Women's Agency. Background paper to Women's Voice, Agency, and Participation (Washington, DC: World Bank, 2013. 
Acceso: 14-01-2016. Disponible en: http://www.worldbank.org/content/dam/Worldbank/document/Gender/ Fleming\%20et\%20al.\%202013\%20Engaging\%20 Men\%20and\%20Boys\%20in\%20Advancing\%20 Women's\%20Agency\%20VAP\%20No.1.pdf.

52. Chant S, Guttman M. Mainstreaming Men into Gender and Development. Oxford, U.K.: Oxfam Great Britain,
2000. Acceso: 16-01-2016. Disponible en: www.oxfa. org.uk/publications

53. Shattuck D, Kerner B, Gilles K, Hartmann M, Ng'ombe $\mathrm{T}$, Guest G. Encouraging Contraceptive Uptake by Motivating Men to Communicate about Family Planning: The Malawi Male Motivator Project. Am J Public Health 2011;101(6):1089-95. 